\title{
Strong Diophantine Triples
}

\author{
Andrej Dujella and Vinko Petričević
}

\section{CONTENTS}

1. Introduction

2. Associated Elliptic Curves

3. Construction of Special Strong Diophantine Pairs and the First Proof of Theorem 1.2

4. The Second Proof of Theorem 1.2

5. "Almost" Strong Diophantine Quadruples Acknowledgments

References
2000 AMS Subject Classification: Primary 11D09; Secondary 11G05, 11Y50

Keywords: Diophantine triples, elliptic curves
We prove that there exist infinitely many triples $a, b, c$ of nonzero rational numbers with the property that $a^{2}+1, b^{2}+1, c^{2}+1$, $a b+1, a c+1$, and $b c+1$ are perfect squares.

\section{INTRODUCTION}

A set $\left\{a_{1}, a_{2}, \ldots, a_{m}\right\}$ of $m$ nonzero integers (rationals) is called a (rational) Diophantine $m$-tuple if $a_{i} \cdot a_{j}+1$ is a perfect square for all $1 \leq i<j \leq m$. Diophantus of Alexandria found a rational Diophantine quadruple $\left\{\frac{1}{16}, \frac{33}{16}, \frac{17}{4}, \frac{105}{16}\right\}$, while the first Diophantine quadruple in integers, the set $\{1,3,8,120\}$, was found by Fermat. Euler was able to add a fifth positive rational, $\frac{777480}{8288641}$, to Fermat's set (for the results of Diophantus, Fermat, and Euler, see [Dickson 66, Diophantus 74, Heath 03]). Euler's construction was generalized in [Dujella 97], where it was shown that every rational Diophantine quadruple the product of whose elements is not equal to 1 can be extended to a rational Diophantine quintuple. Recently, Gibbs [Gibbs 06] found several examples of rational Diophantine sextuples. The first one was

$$
\left\{\frac{11}{192}, \frac{35}{192}, \frac{155}{27}, \frac{512}{27}, \frac{1235}{48}, \frac{180873}{16}\right\} .
$$

A famous conjecture is that there does not exist a Diophantine quintuple (in nonzero integers) (see, for example, [Guy 04, Waldschmidt 04]). In 1969, Baker and Davenport [Baker and Davenport 69] proved that the Fermat set $\{1,3,8,120\}$ cannot be extended to a Diophantine quintuple. In 1998, Dujella and Pethö proved that the pair $\{1,3\}$ cannot be extended to a Diophantine quintuple [Dujella and Pethö 98]. Recently, the first author proved in [Dujella 04] that there does not exist a Diophantine sextuple and that there are only finitely many Diophantine quintuples.

Note that in the definition of (rational) Diophantine $m$-tuples we exclude $i=j$, i.e., the condition that $a_{i}^{2}+1$ is a square. It is obvious that for integers, such a condition makes no sense. But for rationals, there is no obvious 
reason why the sets that satisfy these stronger conditions should not exist. Therefore, we introduce the following notion:

Definition 1.1. A set of $m$ nonzero rationals $\left\{a_{1}, a_{2}, \ldots, a_{m}\right\}$ is called a strong Diophantine $m$-tuple if $a_{i} \cdot a_{j}+1$ is a perfect square for all $i, j=1, \ldots, m$.

It is obvious that there does not exist a strong Diophantine pair consisting of integers. However, it seems to be very hard to find an absolute upper bound for the size of strong (rational) Diophantine tuples. The problem of extension of a given strong Diophantine triple $\left\{a_{1}, a_{2}, a_{3}\right\}$ to a quadruple $\left\{a_{1}, a_{2}, a_{3}, x\right\}$ leads to the hyperelliptic curve

$$
y^{2}=\left(x^{2}+1\right)\left(a_{1} x+1\right)\left(a_{2} x+1\right)\left(a_{3} x+1\right)
$$

of genus $g=2$. According to Lang's conjecture on varieties of general type, there should be an absolute upper bound $B(2, \mathbb{Q})$ for the number of rational points on such curves [Caporaso et al. 97]. This would imply that there does not exist a strong Diophantine $(B(2, \mathbb{Q})+4)$-tuple. However, Lang's conjecture is far from being proved. Let as mention that an example in [Keller and Kulesz 95] shows that $B(2, \mathbb{Q}) \geq 588$. The first strong Diophantine triple, the set

$$
\left\{\frac{1976}{5607}, \frac{3780}{1691}, \frac{14596}{1197}\right\}
$$

was found by the first author in 2000. No example of a strong Diophantine quadruple is known. Although the examples from Section 5 might suggest that the hope of discovering strong Diophantine quadruples is not unrealistic, the existence of a strong Diophantine quintuple is very unlikely.

In [Arkin et al. 93], a family of nonzero rational sextuples $\left\{x_{1}, x_{2}, x_{3}, x_{4}, x_{5}, x_{6}\right\}$ was constructed such that $x_{1}=x_{2}$ and $x_{i} x_{j}+1$ is a perfect square for $i \neq j$. Elements $x_{i}$ were given in terms of Fibonacci numbers. Using the construction from [Dujella 97], we can construct octuples $\left\{x_{1}, x_{2}, x_{3}, x_{4}, x_{5}, x_{6}, x_{7}, x_{8}\right\}$ such that $x_{1}=x_{2}$, $x_{3}=x_{4}, x_{5}=x_{6}$, and $x_{i} x_{j}+1$ is a perfect square for $i \neq j$. For example, we may take

$$
\begin{aligned}
& x_{1}=x_{2}=\frac{1976}{5607}, \quad x_{3}=x_{4}=\frac{3780}{1691}, \\
& x_{5}=x_{6}=\frac{14596}{1197}, \quad x_{7}=\frac{256234396682152}{182474628172489}, \\
& x_{8}=\frac{7374752853358991555754}{1664625949782757005653},
\end{aligned}
$$

or alternatively,

$$
x_{8}=\frac{-429021998726549866}{35408767381264887813} .
$$

We have performed a search for more examples of strong Diophantine triples in various regions. We have found more than 50 such triples with at least two elements with relatively small numerators and denominators. The analysis of the special properties of some of these examples leads us to the following theorem, which is the main result of this paper.

Theorem 1.2. There exist infinitely many strong Diophantine triples of positive rational numbers.

In Sections 3 and 4, we give two different proofs of Theorem 1.2, i.e., two different constructions of infinitely many strong Diophantine triples (and we show that moreover, infinitely many of them have positive elements). Both constructions are based on some elliptic curves over $\mathbb{Q}$ with positive rank.

\section{ASSOCIATED ELLIPTIC CURVES}

To a nonzero rational $a$ we associate the elliptic curve

$$
E_{a}: \quad y^{2}=\left(x^{2}+1\right)(a x+1)
$$

It has a rational point $T=[-1 / a, 0]$, which is the torsion point of order 2 , and another rational point $P=[0,1]$, which is (in general) a point of infinite order. Indeed, by considering the coordinates of the point

$$
3 P=\left[\frac{8 a\left(a^{2}+4\right)}{\left(a^{2}-4\right)^{2}}, \frac{\left(3 a^{2}+4\right)\left(a^{4}+24 a^{2}+16\right)}{\left(a^{2}-4\right)^{3}}\right],
$$

using the Lutz-Nagell theorem, it is easy to check that $P$ has infinite order, except for $a= \pm 2$, when it has order 3. Note that $P+T=\left[a,-a^{2}-1\right]$.

We may consider the elliptic surface $\mathcal{E}$ associated with the family of curves $E_{a}$. We will compute rank $\mathcal{E}(\mathbb{C}(a))$ using Shioda's formula [Shioda 90, Corollary 5.3]:

$$
\operatorname{rank} \mathcal{E}(\mathbb{C}(a))=\operatorname{rank} N S(\mathcal{E}, \mathbb{C})-2-\sum_{\nu}\left(m_{\nu}-1\right) .
$$

Here $N S(\mathcal{E}, \mathbb{C})$ is the Néron-Severi group of $\mathcal{E}$ over $\mathbb{C}$, and the sum ranges over all singular fibers of the pencil $E_{a}$, with $m_{\nu}$ the number of irreducible components of the fiber. Since $\mathcal{E}$ is a rational surface, by [Shioda 90, Lemma 10.1], we have $\operatorname{rank} N S(\mathcal{E}, \mathbb{C})=10$. The numbers $m_{\nu}$ can be easily determined from Kodaira types of singular fibers (see [Miranda 97, Section 4] and [Shioda 
90, p. 224]). The discriminant of $E_{a}$ is $-64 a^{2}\left(a^{2}+1\right)^{2}$, so that $E_{a}$ is singular at $a=0, \pm i, \infty$, and the Kodaira types are $I_{2}, I_{2}, I_{2}$, and $I_{0}^{*}$, respectively. Therefore, we have

$$
\operatorname{rank} \mathcal{E}(\mathbb{C}(a))=10-2-1-1-1-4=1
$$

Since we already know that $[0,1]$ is a point of infinite order on $\mathcal{E}(\mathbb{Q}(a))$, we conclude that here as well, $\operatorname{rank} \mathcal{E}(\mathbb{Q}(a))=1$.

Assume now that $a^{2}+1$ is a perfect square. Then all points of the form $m P$ or $m P+T$ satisfy the additional condition that both factors of the cubic polynomial in $(2-1)$ are perfect squares (by the standard 2-descent argument [Knapp 92, Theorem 4.2 and Proposition 4.6], it suffices to check that this condition is satisfied for $T$, $P$, and $P+T)$. Therefore, the first coordinates of these points induce pairs $\{a, b\}$ that are strong Diophantine pairs. If we parameterize $a$ by $a=\frac{2 t}{t^{2}-1}$, then we may take, for example,

$$
\begin{aligned}
& b=\frac{-\left(t^{2}+t-1\right)\left(t^{2}-t-1\right)}{2 t\left(t^{2}-1\right)}, \\
& b=\frac{t^{6}-1}{2 t^{3}}, \\
& b=\frac{4 t\left(t^{2}-1\right)\left(t^{4}-t^{2}+1\right)}{\left(t^{2}+t-1\right)^{2}\left(t^{2}-t-1\right)^{2}}, \\
& b=\frac{2 t\left(3 t^{4}-t^{8}-1\right)}{\left(t^{2}-1\right)\left(t^{4}+t^{2}+1\right)^{2}},
\end{aligned}
$$

which are respectively the first coordinates of the points $2 P, 2 P+T, 3 P, 3 P+T$.

Assume now that $\{a, b, c\}$ is an arbitrary strong Diophantine triple. Then the points with the first coordinates $b$ and $c$ also belong to $E_{a}(\mathbb{Q})$. Denote these points by $B$ and $C$. Let $e$ and $f$ be the first coordinates of the points $B+T$ and $C+T$, respectively. Then it is easy to verify that $\{a, e, f\}$ is also a strong Diophantine triple. Indeed, we have

$$
\begin{aligned}
e & =\frac{a-b}{a b+1}, & f & =\frac{a-c}{a c+1}, \\
a e+1 & =\frac{a^{2}+1}{a b+1}, & a f+1 & =\frac{a^{2}+1}{a c+1}, \\
e^{2}+1 & =\frac{\left(a^{2}+1\right)\left(b^{2}+1\right)}{(a b+1)^{2}}, & f^{2}+1 & =\frac{\left(a^{2}+1\right)\left(c^{2}+1\right)}{(a c+1)^{2}} .
\end{aligned}
$$

Of course, we can interchange the roles of $a, b, c$ in the above construction. In that way, starting with one strong Diophantine triple $\{a, b, c\}$, we obtain (in general) an- other three strong Diophantine triples:

$$
\begin{aligned}
& \left\{a, \frac{a-b}{a b+1}, \frac{a-c}{a c+1}\right\}, \\
& \left\{b, \frac{b-a}{a b+1}, \frac{b-c}{b c+1}\right\}, \\
& \left\{c, \frac{c-a}{a c+1}, \frac{c-b}{b c+1}\right\} .
\end{aligned}
$$

Note that among these four triples, exactly two have all positive elements (after multiplying all elements by -1 if necessary). Indeed, we may assume that $a>b>c$ and $b>0$. If $c>0$, then exactly

$$
\{a, b, c\} \text { and }\left\{a, \frac{a-b}{a b+1}, \frac{a-c}{a c+1}\right\}
$$

have all positive elements, while if $c<0$, then exactly

$$
\left\{a, \frac{a-b}{a b+1}, \frac{a-c}{a c+1}\right\} \text { and } \quad\left\{-c, \frac{a-c}{a b+1}, \frac{b-c}{b c+1}\right\}
$$

have all positive elements.

Example 2.1. Starting with the triple

$$
\left\{\frac{140}{51}, \frac{187}{84},-\frac{427}{1836}\right\}
$$

we obtain three new strong Diophantine triples:

$$
\begin{aligned}
& \left\{\frac{140}{51}, \frac{2223}{30464}, \frac{278817}{33856}\right\}, \\
& \left\{\frac{187}{84},-\frac{2223}{30464}, \frac{15168}{2975}\right\}, \\
& \left\{\frac{427}{1836}, \frac{278817}{33856}, \frac{15168}{2975}\right\} .
\end{aligned}
$$

However, it should be observed that the four strong Diophantine triples obtained with the above construction are not always necessarily distinct.

Example 2.2. If we start with the triple

$$
\left\{\frac{1976}{5607}, \frac{3780}{1691}, \frac{14596}{1197}\right\},
$$

then the only new triple obtained with the above construction is

$$
\left\{\frac{1976}{5607},-\frac{19853044}{16950717},-\frac{3780}{1691}\right\} .
$$

Note that the strong Diophantine pair

$$
\{a, b\}=\left\{\frac{1976}{5607}, \frac{3780}{1691}\right\}
$$


has the additional property that $a \cdot(-b)+1$ is also a perfect square. The triple

$$
\left\{\frac{1617}{10744}, \frac{15168}{2975}, \frac{99807}{4424}\right\},
$$

that is, its subpair

$$
\left\{\frac{1617}{10744}, \frac{15168}{2975}\right\}
$$

possesses the same property.

In the next section, we will show that there exist infinitely many such pairs.

Lemma 2.3. Each strong Diophantine pair $\{a, b\}$ with the property that $1-a b$ is a perfect square can be extended to a strong Diophantine triple.

Proof: We take $c=\frac{a+b}{1-a b}$, and we claim that $\{a, b, c\}$ is a strong Diophantine triple. Indeed,

$$
\begin{aligned}
a c+1 & =\frac{a^{2}+1}{1-a b}, \\
b c+1 & =\frac{b^{2}+1}{1-a b}, \\
c^{2}+1 & =\frac{\left(a^{2}+1\right)\left(b^{2}+1\right)}{(1-a b)^{2}}
\end{aligned}
$$

are perfect squares.

Remark 2.4. Note that if $c=\frac{a+b}{1-a b}$ (as in the proof of Lemma 2.3), then

$$
\frac{c-a}{a c+1}=b \quad \text { and } \quad \frac{c-b}{b c+1}=a,
$$

and therefore we obtain only two different triples with our construction (only one with positive elements), since in that case

$$
\left\{c, \frac{c-a}{a c+1}, \frac{c-b}{b c+1}\right\}=\{a, b, c\},
$$

while the triples

$$
\left\{a, \frac{a-b}{a b+1}, \frac{a-c}{a c+1}\right\}=\left\{a, \frac{a-b}{a b+1},-b\right\}
$$

and

$$
\left\{b, \frac{b-a}{a b+1}, \frac{b-c}{b c+1}\right\}=\left\{b, \frac{b-a}{a b+1},-a\right\}
$$

are essentially the same (elements of one set are obtained by multiplying the elements of the other set by -1 ). In terms of the elliptic curve $E_{c}$, in this case the addition of the 2-torsion point just interchanges the points with the first coordinates $a$ and $b$.

Example 2.5. Consider the strong Diophantine triple

$$
\left\{\frac{364}{627}, \frac{475}{132},-\frac{132}{475}\right\} .
$$

It has the form $\{a, b,-1 / b\}$. In Section 4 , we will show that there exist infinitely many triples of this form.

Our construction gives now only one new triple,

$$
\left\{\frac{364}{627},-\frac{297}{304}, \frac{304}{297}\right\},
$$

(of the same form). In general, we obtain one new triple

$$
\left\{a, \frac{a-b}{a b+1}, \frac{1+a b}{b-a}\right\}
$$

(and no triples with positive elements). In terms of the elliptic curve $E_{b}$, the point with the first coordinate $c=$ $-1 / b$ is the 2 -torsion point, so in this case, the addition of the 2 -torsion point gives the point at infinity.

\section{CONSTRUCTION OF SPECIAL STRONG DIOPHANTINE PAIRS AND THE FIRST PROOF OF THEOREM 1.2}

In this section, we will first show that there exist infinitely many strong Diophantine pairs $\{a, b\}$ with the additional property that $1-a b$ is also a perfect square.

Hence, we want to find nonzero rationals $a, b$ such that

$$
a^{2}+1, \quad b^{2}+1, \quad a b+1, \quad 1-a b
$$

are perfect squares.

Thus, the question is how we can satisfy the four conditions from (3-1). Let us fix $\alpha:=a \cdot b$ such that $1+\alpha$ and $1-\alpha$ are perfect squares. The condition that $b^{2}+1$ is a square has the parametric solution $b=2 t /\left(t^{2}-1\right)$. Inserting this into the condition that $a^{2}+1$ is a square, we obtain the condition

$$
\alpha^{2}\left(t^{2}-1\right)^{2}+(2 t)^{2}=s^{2} .
$$

The quartic (3-2) can be transformed in the standard way (see, e.g., [Kulesz 03]) into an elliptic curve in Weierstrass form. If such curve has positive rank, we will obtain infinitely many pairs $\{a, b\}$ with the desired property. Let us use the pairs from Example 2.2. For

$$
\alpha=\frac{1617}{10744} \cdot \frac{15168}{2975}=\frac{5544}{7225},
$$


we obtain the curve

$y^{2}+x y=x^{3}-43024332146390 x-32779590846716529900$.

Using a specialized program such as MWRANK [Cremona 97] or APECS [Connell 03], we can compute the rank of this curve. We obtain that the rank is equal to 1 (with the generator $[-802370,-1106521940]$ and torsion group isomorphic to $\mathbb{Z} / 2 \mathbb{Z} \times \mathbb{Z} / 4 \mathbb{Z}$ ). Therefore, we have proved the following lemma.

Lemma 3.1. There exist infinitely many strong Diophantine pairs $\{a, b\}$ with the property that $1-a b$ is a perfect square.

Lemmas 2.3 and 3.1 imply that there exist infinitely many strong Diophantine triples, and by Remark 2.4 we also know that there exist infinitely many such triples with positive elements. Thus, we have actually proved Theorem 1.2. tion:

We list some of the triples obtained with this construc-

$\left\{\frac{54619093071}{22098986000}, \frac{544519015040}{1753879766391}, \frac{107828640285956516216761}{9017829641758970738160}\right\}$,

$\left\{\frac{54619093071}{22098986000},-\frac{544519015040}{1753879766391}, \frac{83762004105751017336761}{68500099164556988313840}\right\}$,

$\left\{\frac{1447635586012047235857910848}{927754486218138903868576025}\right.$,

504261850156211968926214263

$\overline{1025408540091866792066020184}$,

1161361740957008922125901324903233342330112123673647 ).

$\overline{131671608758009932651459660743253005341982491486296}\}$.

For another pair from Example 2.2, i.e.,

$$
\alpha=\frac{1976}{5607} \cdot \frac{3780}{1691}=\frac{6240}{7921}
$$

the rank of the corresponding elliptic curve is equal to 2 , and again we obtain infinitely many strong Diophantine triples. The simplest triple is

$$
\left\{\frac{18685436}{39898077}, \frac{7857720}{4671359}, \frac{400794297231964}{39553316910723}\right\} .
$$

Moreover, we can show that there exist infinitely many $\alpha$ 's with the property that there exist infinitely many rational points on (3-2). Let

$$
a=\frac{u^{2}-1}{2 u}, \quad b=\frac{\left(1+\frac{1}{u}\right)^{2}-1}{2\left(1+\frac{1}{u}\right)}=\frac{2 u+1}{2 u(u+1)} .
$$

Then the conditions that $a b+1$ and $1-a b$ are squares become

$$
\begin{array}{r}
(3 u+1)(2 u-1) \text { is a square, } \\
2 u^{2}+u+1 \text { is a square. }
\end{array}
$$

Multiplying the conditions (3-3) and (3-4) together, we again obtain an elliptic curve. By the transformation $u=\frac{-x-11}{3 x-7}$ we transform it into its Weierstrass form:

$$
y^{2}=x^{3}+37 x+138 .
$$

It has rank equal to 1 , with the generator $P=[-1,10]$ and 2-torsion point $T^{\prime}=[-3,0]$. The point $P$ induces the trivial solution $u=1$ (corresponding to $a=0$ ). By the 2-descent argument, we conclude that the points of the form $(2 k+1) P($ and $(2 k+1) P+T)$ satisfy as well the original system $(3-3),(3-4)$. For example, the point $3 P$ gives the pair

$$
a=\frac{18048}{34655}, \quad b=\frac{12189}{27260},
$$

while the point $5 P$ gives the pair

$$
a=\frac{12423058053504}{12908664457247}, \quad b=\frac{-4521252839715}{14832397620092} .
$$

For each such point, we consider $\alpha=a b=\frac{(u-1)(2 u+1)}{4 u^{2}}$ and the corresponding elliptic curve (3-2). The curve (3-2) has positive rank, since the point $[2 u+1,(2 u+$ $\left.1)\left(u^{2}+1\right) / u\right]$ is of infinite order (for almost all $u$ ). Actually, using Shioda's formula [Shioda 90, Corollary 5.3], it can be proved that the elliptic surface associated with the family of curves (3-2) (which is a K3 surface) has rank over $\mathbb{Q}(u)$ equal to 1 . Therefore, each point on $(3-5)$ of the form $(2 k+1) P$ induces infinitely many strong Diophantine triples by the construction described in Lemma 2.3.

\section{THE SECOND PROOF OF THEOREM 1.2}

In this section we will first prove that there exist infinitely many strong Diophantine triples of the form $\{a, b,-1 / b\}$.

Assume that we can somehow find nonzero rationals $b, g$ such that

$$
b^{2}+1, \quad g^{2}+1, \quad \frac{b}{g}, \quad \frac{b}{g}+1, \quad \frac{g}{b}+1
$$

are perfect squares. Define $a=\frac{b g-1}{b+g}$. Then we claim that $\{a, b,-1 / b\}$ is a strong Diophantine triple. Indeed, we have

$$
\begin{aligned}
& a b+1=\frac{g\left(b^{2}+1\right)}{b+g}, \quad a \cdot\left(-\frac{1}{b}\right)+1=\frac{b^{2}+1}{b(b+g)}, \\
& a^{2}+1=\frac{\left(b^{2}+1\right)\left(g^{2}+1\right)}{(b+g)^{2}}
\end{aligned}
$$

Let us fix a positive rational $\beta$ such that $\beta^{2}+1$ is a perfect square. If $b / g=\beta^{2}$, then the last three conditions 
from (4-1) are satisfied. The remaining conditions are that $g^{2}+1$ and $\beta^{4} g^{2}+1$ are perfect squares. Multiplying these two conditions together, we again obtain an elliptic curve, and we hope that it will have positive rank. So, let us use Example 2.5, i.e., put

$$
\beta^{2}=\frac{475}{132} \cdot \frac{297}{304}=\left(\frac{15}{8}\right)^{2} .
$$

We obtain the quartic

$$
z^{2}=\frac{50625}{4096} g^{4}+\frac{54721}{4096} g^{2}+1
$$

which we transform (with $g=(128 x+1167392) /(2 y+x)$ ) into minimal Weierstrass form:

$$
E: \quad y^{2}+x y=x^{3}-114223080 x-283150929600 .
$$

Using MWRANK we find that this curve has rank equal to 1 , with generator $Q=[-8520,-263280]$ and torsion group $\mathbb{Z} / 2 \mathbb{Z} \times \mathbb{Z} / 4 \mathbb{Z}$ generated by $T_{1}=[29100,4571880]$ and $T_{2}=[-36481 / 4,36481 / 8]$.

We are interested in rational points on this curve for which the corresponding number $g$ satisfies the condition that $g^{2}+1$ is a perfect square. We have

$$
g^{2}+1=(4 x+36481)(x-2512)^{2} .
$$

We note here the linear factor $4 x+36481$, which corresponds to the 2 -torsion point $T_{2}$. Since all points from $E(\mathbb{Q}) / 2 E(\mathbb{Q})$ satisfy the condition that $g^{2}+1$ is a perfect square, by the 2-descent argument used already several times in this paper, this condition is satisfied for all points in $E(\mathbb{Q})$. Therefore, we have proved the following lemma.

Lemma 4.1. There exist infinitely many strong Diophantine triples of the form $\{a, b,-1 / b\}$.

Torsion points induce trivial solutions with $a=0$. The point $P$ induces

$$
g=-\frac{28}{195}, \quad b=-\frac{105}{208}, \quad a=\frac{37620}{26299},
$$

which gives the triple

$$
\left\{\frac{37620}{26299},-\frac{105}{208}, \frac{208}{105}\right\} \text {. }
$$

Some other triples obtained with the this construction (for points of the form $i P+T^{\prime \prime}$, where $i=1,2$ and $T^{\prime \prime}$ is a torsion point) are

$$
\begin{aligned}
& \left\{\frac{364}{627},-\frac{297}{304}, \frac{304}{297}\right\}, \\
& \left\{\frac{37620}{26299}, \frac{195}{28},-\frac{28}{195}\right\},
\end{aligned}
$$

$$
\begin{aligned}
& \left\{\frac{232371144612352}{548740392625425},-\frac{4176991}{3636600}, \frac{3636600}{4176991}\right\}, \\
& \left\{\frac{28481335257375}{14523196538272},-\frac{9106080}{23923351}, \frac{23923351}{9106080}\right\} .
\end{aligned}
$$

Of course, triples of the form $\{a, b,-1 / b\}$ cannot have all positive elements. We will now describe how from a triple of the form $\{a, b,-1 / b\}$, a new strong Diophantine triple with positive elements can be constructed. This will also give a connection between Diophantine triples of the form $\{a, b,-1 / b\}$ and the special Diophantine pairs from Section 3 .

Let $\{a, b,-1 / b\}$ be a strong Diophantine triple, and define $g=(a b+1) /(b-a)$. We may assume that $a$ and $b$ are positive. The product $b g=\frac{a b+1}{1-a / b}$ is a perfect square, so there exists a rational number $t>1$ such that $b g=t^{2}$. Let us define $h=\frac{2 t}{t^{2}-1}$. Then $h$ is positive and $h^{2}+1$ is a perfect square. Moreover, we will show that $a h+1$ and $1-a h$ are perfect squares. We have

$$
a=\frac{b\left(t^{2}-1\right)}{b^{2}+t^{2}} \text {. }
$$

Hence,

$$
b^{2}+t^{2}=\left(t^{2}-1\right) \cdot \frac{b}{a}=\frac{b^{2}+1}{1-a / b}
$$

is a perfect square. Therefore,

$$
a h+1=\frac{(t+b)^{2}}{b^{2}+t^{2}}, \quad 1-a h=\frac{(t-b)^{2}}{b^{2}+t^{2}},
$$

are also perfect squares. Now we can apply the construction from Example 2.2, and we obtain the strong Diophantine triple

$$
\left\{a, h, \frac{a+h}{1-a h}\right\}
$$

with positive elements. This construction, together with Lemma 4.1, gives a new proof of Theorem 1.2.

For example, starting with the triple

$$
\left\{\frac{37620}{26299}, \frac{195}{28},-\frac{28}{195}\right\}
$$

we obtain the triple

$$
\left\{\frac{37620}{26299}, \frac{364}{627}, \frac{33160576}{2795793}\right\} .
$$

\section{5. "ALMOST" STRONG DIOPHANTINE QUADRUPLES}

It is not known whether there exist any strong Diophantine quadruples. Such a set has to satisfy ten conditions 
of the form $x y+1$ is a square. However, we were able to find quadruples (with relatively small numerators and denominators) satisfying nine of these ten conditions. In Example 2.1, we considered the strong Diophantine triple $\left\{\frac{140}{51}, \frac{187}{84},-\frac{427}{1836}\right\}$. Perhaps surprisingly, we were able to find another extension of the pair $\left\{\frac{140}{51}, \frac{187}{84}\right\}$ to a strong Diophantine triple, namely the triple $\left\{\frac{140}{51}, \frac{187}{84},-\frac{7200}{20111}\right\}$. Therefore, we obtained an "almost" strong Diophantine quadruple

$$
\left\{\frac{140}{51}, \frac{187}{84},-\frac{427}{1836},-\frac{7200}{20111}\right\},
$$

which satisfies almost all conditions for a strong Diophantine quadruple. The only missing condition comes from the fact that $\left(-\frac{427}{1836}\right) \cdot\left(-\frac{7200}{20111}\right)+1$ is not a perfect square.

Using the construction from Section 3, we can find another example with the same property (and with positive elements):

$$
\left\{\frac{140}{51}, \frac{2223}{30464}, \frac{278817}{33856}, \frac{3182740}{17661}\right\} .
$$

In this case, the only missing condition is that $\frac{278817}{33856}$. $\frac{3182740}{17661}+1$ is not a perfect square.

\section{ACKNOWLEDGMENTS}

The first author was supported by the Ministry of Science, Education and Sports, Republic of Croatia, grant 037-03727812821.

\section{REFERENCES}

[Arkin et al. 93] J. Arkin, D. C. Arney, F. R. Giordano, R. A. Kolb, and G. E. Bergum. "An Extension of an Old Classical Diophantine Problem." In Application of Fibonacci Numbers, Vol. 5, edited by G. E. Bergum, A. N. Philippou, and A. F. Horadam, pp. 45-48. Dordrecht: Kluwer, 1993.

[Baker and Davenport 69] A. Baker and H. Davenport. "The Equations $3 x^{2}-2=y^{2}$ and $8 x^{2}-7=z^{2}$." Quart. J. Math. Oxford Ser. (2) 20 (1969), 129-137.

[Caporaso et al. 97] L. Caporaso, J. Harris, and B. Mazur. "Uniformity of Rational Points." J. Amer. Math. Soc. 10 (1997), 1-35.
[Connell 03] I. Connell. "APECS." Available online (ftp:// ftp.math.mcgill.ca/pub/apecs/), 2003.

[Cremona 97] J. Cremona. Algorithms for Modular Elliptic Curves. Cambridge: Cambridge University Press, 1997.

[Dickson 66] L. E. Dickson. History of the Theory of Numbers, Vol. 2. New York: Chelsea, 1966.

[Diophantus 74] Diophantus of Alexandria. Arithmetic and the Book of Polygonal Numbers (in Russian), edited by I. G. Bashmakova. Moscow: Nauka, 1974.

[Dujella 97] A. Dujella. "On Diophantine Quintuples." Acta Arith. 81 (1997), 69-79.

[Dujella 04] A. Dujella. "There Are Only Finitely Many Diophantine Quintuples." J. Reine Angew. Math. 566 (2004), $183-214$.

[Dujella and Pethő 98] A. Dujella and A. Pethő. "A Generalization of a Theorem of Baker and Davenport." Quart. J. Math. Oxford Ser. (2) 49 (1998), 291-306.

[Gibbs 06] P. Gibbs. "Some Rational Diophantine Sextuples." Glas. Mat. Ser. III 41 (2006), 195-203.

[Guy 04] R. K. Guy. Unsolved Problems in Number Theory, 3rd edition. New York: Springer-Verlag, 2004.

[Heath 03] T. L. Heath. Diophantus of Alexandria. A Study of the History of Greek Algebra (Cambridge, 1910), Powell's Bookstore, Chicago; Martino Publishing, Mansfield Center, 2003.

[Keller and Kulesz 95] W. Keller and L. Kulesz. "Courbes algébriques de genre 2 and 3 posśedant de nombreux points rationnels." C. R. Acad. Sci. Paris Sér. I 321 (1995), 14691472 .

[Knapp 92] A. Knapp. Elliptic Curves. Princeton: Princeton Univ. Press, 1992.

[Kulesz 03] L. Kulesz. "Families of Elliptic Curves of High Rank with Nontrivial Torsion Group over $\mathbb{Q}$." Acta Arith. 108 (2003), 339-356.

[Miranda 97] R. Miranda. "An Overview of Algebraic Surfaces." In Algebraic Geometry (Ankara, 1995), pp. 157-217, Lecture Notes in Pure and Appl. Math. 193. New York: Dekker, 1997.

[Shioda 90] T. Shioda. "On the Mordell-Weil Lattices." Comment. Math. Univ. St. Pauli 39 (1990), 211-240.

[Waldschmidt 04] M. Waldschmidt. "Open Diophantine Problems." Moscow Math. J. 4 (2004), 245-305.

Andrej Dujella, Department of Mathematics, University of Zagreb, Bijenička cesta 30, 10000 Zagreb, Croatia (duje@math.hr)

Vinko Petričević, Department of Mathematics, University of Zagreb, Bijenička cesta 30, 10000 Zagreb, Croatia (vpetrice@math.hr)

Received October 21, 2006; accepted September 22, 2007. 